\title{
FOURIER TRANFORMS IN GENERALIZED FOCK SPACES
}

\author{
JOHN SCHMEELK \\ Department of Mathematical Sciences \\ Box 2014, Oliver Hall, 1015 W. Main Street \\ Virginia Commonwealth University \\ Richmond, VA 23284-2014 \\ (Rocoived June 16, 1989)
}

ABSTRACT, A classical Fock space consists of functions of the form,

$$
\Phi \leftrightarrow\left(\phi_{0}, \phi_{1}, \ldots, \phi_{q}, \ldots\right),
$$

where $\phi_{0} \varepsilon C$ and $\phi_{q} \varepsilon L^{2}\left(R^{3 q}\right), q>1$. We will replace the $\phi_{q}, q>1$ with

q-symmetric rapid descent test functions within tempered distribution theory. This space is a natural generalization of a classical Fock space as seen by expanding functionals having generalized Taylor series. The particular coefficients of such series are multilinear functionals having tempered distributions as their domain. The Fourier transform will be introduced into this setting. A theorem will be proven relating the convergence of the tranform to the parameter, $s$, which sweeps out a scale of genralized Fock spaces.

KEY WORDS AND PHRASES. Generalized Fock Sapces, tempered distibutions, Fourier transforms, and rapid descent test functions.

1980 AMS SUBJECT CLASSIFICATION CODE. Primary 46FXX Secondary $44 A 15$

1. INTRODUCTION.

Rapid descent test functions, $S\left(R^{q}\right)$, and their dual tempered distributions, $S^{\prime}\left(R^{q}\right)$, are excellent spaces to do the analysis of the Fourier transform (Bogolubov and Logunov [1], Constantinescu [2], Freidman [3], Ge1fand and Shilov [4], and Lighthill [5]). The classical Fourier transform analysis examines spaces having test functions defined on a finite number of independent variables. By this we mean the independent variables of a rapid descent test function, $\phi\left(t_{1}, \ldots t_{q}\right)$, belonging to a $q-$ dimensional Euclidean space. This paper will indicate a method that will enjoy the property that the number of independent variables becomes infinite, that is in some sense the dimension, $q \rightarrow \infty$. The need for this analysis is essential in advanced physics. An infinite number of particles are described by state vectors in a Fock space. The classical results are developed in a Hilbert space. Traditionally the Lebseque integrable functions, $L^{p}\left(R^{q}\right)$, are implemented in the construction of a direct 
sum of these spaces. However, when you want to describe a frequency of a parlicie lhe Fourier transform must be studied. This presents a significant problem since the kerne1, $e^{-2 \pi i t w}$, does not belong to any $L^{p}\left(R^{q}\right)$ space. This kernel problem is solved in tempered distribution theory (Constantinescu [2], Gelfand and Shilvo [4], Lighthi11 [5], and Zemanian [6]) but the infinite number of variables problem still remains. This paper will implement tempered distributions together with a holomorphic functional theory developed in Schmeelk [7-10] to solve the infinite number of variables problem.

We briefly recall in $S\left(R^{q}\right)$ and $S^{\prime}\left(R^{q}\right)$ the Fourier transforms are respectively defined as

$$
(F \phi)\left(w_{1} \ldots w_{q}\right) \triangleq \int_{R} q \exp -2 \pi\left[t_{1} w_{1}+\ldots+t_{q} w_{q} \phi\left(t_{1} \ldots t_{q}\right) d t_{1} \ldots d t q\right.
$$

and

$$
\left\langle F \quad F\left(w_{1} \ldots w_{q}\right), \phi\left(t_{1} \ldots t_{q}\right)\right\rangle \triangleq\left\langle F\left(w_{1} \ldots w_{q}\right), F\left(\phi\left(t_{1} \ldots t_{q}\right)\right)\right\rangle
$$

for all $\phi\left(t_{1} \ldots t_{q}\right)$ a $\varepsilon S\left(R^{q}\right)$ and all $\mathbf{F}\left(w_{1} \ldots w_{q}\right) \varepsilon S^{\prime}\left(R^{q}\right)$. The advantages of $S\left(R^{q}\right)$ and $S^{\prime}\left(R^{q}\right)$ are many but the fundamental result is that the Fourier transform exists a homeomorphism and has the appropriate derivative - multiplication property. This paper will not include a survey of the many Fourier transform properties which are contained in Constantinescu [2], Friedman [3], Zemanian [6], Bracewe11 [11], Gonzalez and Wintz [12], and Papoulis [13].

We will extend the Fourier transform into generalized Fock spaces. The principle result will be the existence of the transform in the scale of Frechet spaces

$\Gamma^{\mathrm{PB}}=\bigcup_{\mathrm{s}} \Gamma^{\mathrm{p}, \mathrm{sB}}$ and its corresponding dual, $\left(\Gamma^{\mathrm{pB}}\right)^{\prime} \cdot$ A comprehensive examination of these spaces are contained in Schmeelk [7-10]. We will only review these spaces in sections 2 and 3 .

2. THE SPACE, $\Gamma^{\mathrm{pB}}=\bigcup_{s} \Gamma_{1}^{\mathrm{P}, \mathrm{sB}}$

For each $s>1$, the space $\Gamma^{p, s B}\left(p>1, B=\left\{B_{i}\right\}_{i=0}^{\infty}, B_{1}>B_{j}, f>1\right)$, is called an infinite dimensional Fock space. Then $p$ and $B_{1}, 1>0$ are all real numbers. These spaces are topological spaces of complex valued functionals on $S^{\prime}(R ; C)$, the space of complex valued distributions. The functionals which are members of $\Gamma^{\mathrm{p}, \mathrm{sB}}$ are all

$C^{\infty}\left(S^{\prime}(R) ; \mathbb{C}\right)$. The complex or real valued functionals enjoy similar properties. The

real valued functionals which are members of $\Gamma^{\mathrm{PB}}$ are developed in Schmeelk [8].

We also require if $\Phi \in \Gamma^{\mathrm{p}, \mathrm{sB}}$, then

$$
\Phi(x)=\sum_{q=0}^{\infty} a_{q} x^{q}=\sum_{q=0}^{\infty} a_{q}[x, \ldots, x]
$$

where $a_{0} \varepsilon \mathbb{C}$ and $a_{q}, q>1$ are $q$-multilinear symmetric continuous functionals on the space, $S^{\prime}(R) x_{0} \ldots S^{\prime}(R),(q$ copies, $q>1)$ to $\mathbb{C}$. We identify for each $\Phi \varepsilon I^{p, s B}$ the 
associated Fock state vector,

$$
\leftrightarrow \leftrightarrow\left[\begin{array}{c}
a_{0} \\
a_{1} \\
\vdots \\
a_{q} \\
\vdots
\end{array}\right]
$$

We equip our infinite dimensional Fock vector space with the following increasing sequence of norms:

where

$$
\|\| \Phi \|_{s B_{m}}=\sup _{q} \frac{\left\|a_{q}\right\|_{m} q !^{1 / p}}{\left(s B_{m}\right)^{q}}, m=0,1, \ldots
$$

with

$$
\left\|a_{q}\right\|_{m}=\sup _{\|x\|}\left|a_{q_{m}} x^{q}\right|, x \in S^{\prime}(R), m=0,1, \ldots
$$

$$
\left\|\left|x \|_{-m}=\sup _{\|\phi\|_{m} \leqslant 1}\right|\langle x, \phi\rangle \mid, \phi \varepsilon S(R), m=0,1 \ldots\right.
$$

and

where

$$
\begin{aligned}
\|\phi\|_{m}= & \sup _{0<\alpha_{1}<m} M_{m}\left(t_{1}, \ldots, t_{q}\right) \mid D \alpha_{\phi\left(t_{1} \ldots t_{q}\right) \mid} \\
& 1<1<q \\
& \left(t_{1}, \ldots, t_{q}\right) \in R^{q}
\end{aligned}
$$

and

$$
M_{m}\left(t_{1}, \ldots, t_{q}\right) \triangleq\left[\left(1+\left(2 \pi t_{1}\right)^{2}\right) \ldots\left(1+\left(2 \pi t_{q}\right)^{2}\right)\right]^{m}
$$

$$
D^{\alpha}=\frac{\partial \alpha_{1}+\ldots+\alpha_{q}}{\partial t_{1}^{\alpha_{1}} \ldots \partial t_{q}^{\alpha}}
$$

The norms defined in expression (2.6) using the functions $M_{m}\left(t_{1}, \ldots, t_{q}\right)$ so defined generate a sequence of norms equivalent to the sequence of norms implementing the functions, $\dot{M}_{m}^{\prime}\left(t_{1}, \ldots, t_{q}\right)=\left[\left(1+\left|t_{1}\right|\right) \ldots\left(1+\left|t_{q}\right|\right)^{m},[2,3]\right.$.

It was proven in reference $[10]$ that each real valued functional, $\Phi \varepsilon \Gamma^{p, s B}$, has a kernel representation which remains valid for complex valued functionals. This representation is as follows,

$$
\Phi \leftrightarrow\left[\begin{array}{c}
\phi_{0} \\
\phi_{1} \\
\vdots \\
\phi_{q}\left(t_{1}, \ldots, t_{q}\right)
\end{array}\right]
$$

where $\phi_{0}=a_{0}$ and $\phi_{q}\left(t_{1}, \ldots, t_{q}\right)$ are symmetric complex valued rapid descent test functions, $S_{+}\left(R^{q}\right)$ satisfying,

where

$$
\|\Phi\|_{s B_{m}}=\sup _{q} \frac{\left\|\phi_{q}\right\|_{m} q !^{1 / p}}{\left(s B_{m}\right)}, m=0,1, \ldots
$$


434

J. SCHMEELK

$$
\begin{aligned}
\left\|\phi_{q}\right\|_{m}= & \sup _{0} \alpha_{1}<m \\
1<1<q & M_{m}\left(t_{1}, \ldots, t_{q}\right)\left|D^{\alpha} \phi\left(t_{1} \ldots t_{q}\right)\right| . \\
& \left(t_{1}, \ldots t_{q}\right) \in R^{q}
\end{aligned}
$$

The representation for $\Phi$ given in expression (2.8) enjoys the standard square summable property often times postulated for rock functionals as seen by the following theorem.

THEOREM 2.10. Given a $\Phi \varepsilon \Gamma^{\mathrm{p}, \mathrm{sB}}$, its kernel representation given in expression (2.8) satisfies

$$
|| \Phi||_{L_{2}}=\left|\phi_{0}\right|^{2}+\sum_{q=1}^{\infty} \int_{R^{q}}\left|\phi_{q}\left(t_{1}, \ldots, t_{q}\right)\right|^{2} d t_{1}, \ldots, d t_{q}<\infty .
$$

PROOF. Clearly the constant, $\left|\phi_{0}\right|^{2}$, does not contribute to the convergence problem of the result of the theorem. Also since $\Phi \in \Gamma^{p, s B}$, then by the requirement given in expression (2.9) there must exist. a sequence of positive constants, $\left\{C_{m}\right\}_{m=0}^{\infty}$, such that

$$
\sup _{q}\left\|\phi_{q}\right\|_{m}<\frac{C_{m}\left(s_{m}\right)^{q}}{q !^{1 / p}}
$$

for $a 11 \mathrm{q}$ and $m=0,1, \ldots$. We now consider a partial sum,

$$
\begin{aligned}
& \sum_{q=1}^{q_{R} q} \int_{q}\left|\phi_{q}\left(t_{1}, \ldots, t_{q}\right)\right|^{2} d t_{1} \ldots d t_{q} \\
& =\frac{\left[\sum_{q=1}^{q_{0}} \int_{R} \frac{M_{2 m}\left(t_{1}, \ldots, t_{q}\right)}{M_{2 m}\left(t_{1}, \ldots, t_{q}\right)}\left|\phi_{q}\left(t_{1}, \ldots, t_{q}\right)\right|^{2} d t_{1} \ldots t_{q}\right] q !^{\left.1 / p_{(s B}\right)^{q}}}{q !^{\left.1 / p_{(s B}\right)^{q}}} \\
& <\frac{\sum_{q=1}^{q_{0}}\left\|\phi_{q}\right\|_{m}^{2}\left[\int_{R} \frac{1}{M_{2 m}\left(t_{1}, \ldots, t_{q}\right)} d t_{1} \ldots d t_{q}\right] q !^{\left.1 / p_{(s B}\right)^{q}}}{q !^{1 / p_{\left(s B_{m}\right)^{q}}}} \\
& <\sum_{q=1}^{q_{0}} \frac{\left\|\phi_{q}\right\|_{m}^{2} \pi^{q} q !^{1 / p}\left(s B_{m}\right)^{q}}{q !^{2 / p}\left(s B_{m}\right)^{q}} \\
& =\sum_{q=1}^{q} \frac{\left\|\phi_{q}\right\|_{m} q !^{1 / p}}{\left(s B_{m}\right)^{q}} \frac{\left\|\phi_{q}\right\|_{m} \pi^{q}\left(s B_{m}\right)^{q}}{q !^{1 / p}} \\
& <\|\| \Phi \|_{s B_{m}} \sum_{q=1}^{q_{0}} \frac{\left\|\phi_{q}\right\|_{m}^{2} \pi^{q}\left(s B_{m}\right)^{q}}{q !^{1 / p}} \\
& <\|\| \Phi \|{ }_{s B} \sum_{m=1}^{q_{0}} \frac{C_{m} \pi^{q}\left(s B_{m}\right)^{2 q}}{q !^{2 / p}}<\infty .
\end{aligned}
$$

Since expression (2.11) converges for any $q_{0}$, the result follows. 
3. THE FOURIER TRANSFORM IN $\mathrm{I}^{\mathrm{pB}}$.

DEFINITION 3.1. The Fourier transform $\mathcal{F}$ on $\Phi \in \mathrm{r}^{\mathrm{pB}}$ is defined as follows,

$$
\mathcal{F}:\left[\begin{array}{c}
\phi_{0} \\
\phi_{1} \\
\vdots \\
\phi_{q} \\
\vdots
\end{array}\right]
$$

$$
[
$$

$$
\int_{R_{1}}^{\phi_{0}} \exp \left[-2 \pi i t_{1} w_{1}\right] \phi_{1}\left(t_{1}\right) d t
$$

LEMMA 3.2. $\mathcal{F}(\Phi)$ is well defined for every $\Phi \varepsilon \mathrm{r}^{\mathrm{p}, \mathrm{sB}}$ and moreover

$$
\phi_{0}+\sum_{q=1}^{\infty} \int_{R} q
$$

PROOF. $\Phi \varepsilon \mathrm{r}^{\mathrm{PB}}$ implies $\Phi \varepsilon \mathrm{r}^{\mathrm{p}, \mathrm{sB}}$ for some $s>1$.

We then have

$$
\begin{aligned}
& \phi_{0}+\left|\sum_{q=1}^{\infty} \int_{R}^{q}\left[\exp -2 \pi i\left[t_{1} w_{1}+\ldots+t_{q} w_{q}\right]\right]_{q}\left(t_{1}, \ldots, t_{q}\right) d t_{1} \ldots d t_{q}\right|<\infty . \\
& <\phi_{0}+\left|\sum_{q=1}^{\infty} \int_{R^{q}} \frac{M_{1}\left(t_{1}, \ldots, t_{q}\right)}{M_{1}\left(t_{1}, \ldots, t_{q}\right)}\right| \phi_{q}\left(t_{1}, \ldots, t_{q}\right) \mid d t_{1}, \ldots, d t_{q} \\
& <\left|\phi_{0}\right|+\sup _{\left(t_{1}, \ldots, t_{q}\right) \in R^{q}} M_{1}\left(t_{1}, \ldots, t_{q}\right)\left|\phi_{q}\left(t_{1}, \ldots, t_{q}\right)\right| \sum_{q=1}^{\infty} \int_{R^{q}} \frac{1}{M_{1}\left(t_{1}, \ldots, t_{q}\right)} d t_{1} \ldots d t q \\
& <\left|\phi_{0}\right|+\sum_{q=1}^{\infty} \frac{\pi^{q}\left\|\phi_{q}\right\|_{1} q !^{1 / p}\left(s B_{m}\right)^{q}}{q !^{1 / p}\left(s B_{m}\right)^{q}} \\
& <\left|\phi_{0}\right|+\|\| \Phi \mid \|_{s B_{m}} \sum_{q=1}^{\infty} \frac{\pi^{q}\left(s B_{m}\right)^{q}}{q !^{1 / p}}<\infty
\end{aligned}
$$

THEOREM 3.4. The Fourier transform is a linear continuous transformation on $r^{\mathrm{pB}}$ to $\mathrm{I}^{\mathrm{PB}}$. PROOF. Since $r^{\mathrm{pB}}=\bigcup_{S} \Gamma^{\mathrm{p}, \mathrm{sB}}$, we consider the Fourler transform on the space, $r^{p, s B}$, to the space, $r^{p}, s^{\prime B}$, where $s^{\prime}>s \pi$. We have for any norm \|\|$\cdot\|\|_{s} B_{m}$, the following,

$$
\left\|\mathcal{F}_{\Phi}\right\|_{s^{\prime} B_{m}}=\frac{\left\|\int^{q} \exp \left[-2 \pi i\left(t_{1} w_{1}+\ldots+t_{q} w_{q}\right)\right] \phi\left(t_{1}, \ldots, t_{q}\right) d t_{1} \ldots, d t_{q}\right\| \|_{m} q !^{1 / p}}{\left(s^{\prime} B_{m}\right)^{q}}
$$




$$
\begin{aligned}
& \mid D^{\alpha} \int_{R} \exp \left(\left[-2 \pi i\left(t_{1} w_{1}+\ldots+t_{q} w_{q}\right)\right] \phi\left(t_{1}, \ldots, t_{q}\right) d t_{1} \ldots d t_{q} \mid q !^{1 / p}\right. \\
& =\sup _{q} M_{m}\left(w_{1}, \ldots, w_{q}\right)-\frac{R^{q}}{\left(s^{\prime} B_{m}\right)^{q}} \\
& 0<\alpha_{1}<m \\
& 1<\mathrm{i}<\mathrm{q} \\
& \left(w_{1}, \ldots, w_{q}\right) \in R^{q} \\
& =\sup _{q} M_{m}\left(w_{1}, \ldots, w_{q}\right) \\
& 0<\alpha<m \\
& 1<1<q \\
& \left(w_{1}, \ldots, w_{q}\right) \in R^{q} \\
& \frac{\int_{R} \int_{q}\left(-2 \pi i t_{1}\right){ }^{\alpha} \ldots\left(-2 \pi i t_{q}\right) \stackrel{\alpha}{q_{q}} \exp \left[-2 \pi i\left(t_{1} w_{1}+\ldots+t_{q} w_{q}\right)\right] \phi\left(t_{1}, \ldots, t_{q}\right) d t_{1} \ldots d t_{q} \mid q !^{1 / p}}{\left(s^{\prime} B_{m}\right)^{q}} \\
& \leqslant \sup _{q} \frac{M_{m}\left(w_{1}, \ldots w_{q}\right) \int_{R} q_{m} M_{m}\left(t_{1}, \ldots, t_{q}\right) \mid \phi\left(t_{1}, \ldots, t_{q}\right) / d t_{1} \ldots d t q q !^{1 / p}}{\left(s^{\prime} B_{m}\right)^{q}} \\
& 0<\alpha_{1}<m \\
& \begin{array}{c}
1<1<q \\
\left(w_{1}, \ldots, w_{q}\right) \in R^{q}
\end{array} \\
& \sup _{q} \frac{\int_{R^{q}} M_{2 m}\left(t_{1}, \ldots, t_{q}\right)\left|\phi\left(t_{1}, \ldots, t_{q}\right)\right| d t_{1} \ldots d t_{q}}{\left(s_{m}^{\prime} B^{q}\right)} q ! 1 / p \\
& 1<1<q
\end{aligned}
$$$$
\sup _{0<\alpha_{1}<m} \frac{\int_{q}^{q} \frac{M_{2 m+1}\left(t_{1}, \ldots, t_{q}\right)}{M_{1}\left(t_{1}, \ldots, t_{q}\right)}\left|\phi\left(t_{1}, \ldots, t_{q}\right)\right| d t_{1} \ldots d t_{q}}{\left(s^{\prime} B_{m}\right)^{q}} q !^{1 / p}
$$$$
1<1<q
$$$$
=\sup _{\substack{q}} \frac{M_{2 m+1}\left(t_{1}, \ldots, t_{q}\right)\left|\phi\left(t_{1}, \ldots, t_{q}\right)\right| \pi^{q} q !^{1 / p}}{\left(s^{\prime} B_{m}\right)^{q}}
$$$$
\left(t_{1}, \ldots, t_{q}\right) \in R^{q}
$$ 


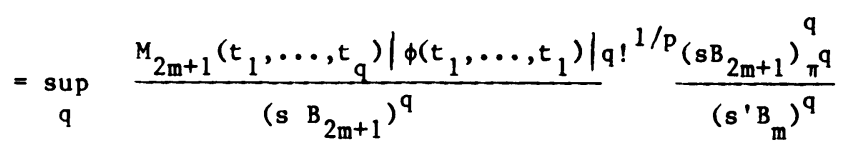

$$
\begin{aligned}
& 0<\alpha_{1}<m \\
& 1<1<q \\
& \left(t_{1}, \ldots, t_{q}\right) \in R^{q} \\
& <\sup _{q} \frac{\|\phi\|_{2 m+1} q !^{1 / p}}{\left(s_{2 m+1}\right)^{q}}\left(\frac{\left(s B_{2 m+1}\right) \pi}{s^{\prime} B_{m}}\right)^{q} .
\end{aligned}
$$

Noting that $B_{2 m+1}\left\langle B_{m}\right.$ and $s^{\prime}>s \pi$ implies expression (3.5) is finite.

4. THE FOURIER TRANSFORM ON $\left(\Gamma^{\mathrm{pB}}\right)^{\prime}$

In a previous paper [9], it was shown that the dual of $\Gamma^{p, s B}$ denoted $\left(\Gamma^{p, s B}\right)^{\prime}$ is the union of sets of the form,

$$
\begin{aligned}
& \left(\Gamma_{-m}^{p, s B}\right)=\left\{\left(F_{0}, F_{1}, \ldots, F_{q}, \ldots\right): F_{0} \in C,\right. \\
& \left.F_{q} \varepsilon S_{R}^{\prime}+\left(R^{q}\right), \sum_{q=0}^{\infty}\left\|F_{q}\right\|_{-m}\left(s B_{m}\right)^{q} q !-1 / p<\infty\right\} .
\end{aligned}
$$

The generalized Fock dual functionals described in expression (4.1) can also be considered as sequences where the $F_{q}$ are symmetric tempered distributions all having rank <m. We also note if $\Phi \in \Gamma^{p, s B}$ and $F \varepsilon\left(\Gamma^{p, s B}\right)$, then the evaluation of $F$ at $\$$ is denoted as

$$
\left.\langle\langle F, \Phi\rangle\rangle \triangleq \sum_{q=0}^{\infty} F_{q}, \phi_{q}\right\rangle \text {. }
$$

EXAMPLE. 4.3 All the sets, $\left(\Gamma_{-m}^{p, 8 B}\right)$, contain the generalized Fock Dirac functiona1,

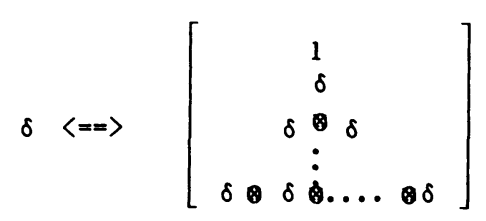

where $\delta \delta \ldots \delta$ is the tensor product of $q$ copies of the Dirac delta functional [3]. We immediately verify that

$$
\begin{aligned}
\|\delta\|_{-\left(s B_{m}\right)} & \triangleq \sum_{q=0}^{\infty}\left\|F_{q}\right\|_{-m}\left(s B_{m}\right)^{q} q !^{-1 / p} \\
& =\sum_{q=0}^{\infty}\|\delta \otimes \ldots \delta \delta\|_{-m}\left(s B_{m}\right)^{q} q !^{-1 / p} \\
& \leqslant \sum_{q=0}^{\infty} 1 \cdot\left(s B_{m}\right)^{q} q !^{-1 / p}<\infty .
\end{aligned}
$$


DEFINITION 4.4. The Fourier Lransform on the space $\left(\Gamma^{\mathrm{pB}}\right)$ ' is defined a;

$$
\left\langle\left\langle\xi_{F}, \Phi\right\rangle\right\rangle \Delta\left\langle\left\langle F, \xi_{\Phi}\right\rangle\right\rangle \text {. }
$$

EXAHPLE 4.4. We compute the Fourier transform of

$$
\delta^{(k)}(t-\tau) \Leftrightarrow\left[\begin{array}{c}
1 \\
\delta^{(k)}\left(t_{1}-\tau_{1}\right) \\
\vdots \\
\delta^{(k)}\left(t_{1}-\tau_{1}\right) \\
\delta^{(k)}\left(t_{1}-\tau_{1}\right) \ldots \delta^{(k)}\left(t_{q}-\tau_{2}\right) \\
\vdots
\end{array}\right]
$$

It suffices to consider the $q^{\text {th }}$ component,

$$
\begin{aligned}
& \left\langle\mathcal{F} \delta^{(k)}\left(t_{1}-\tau_{1}\right) \otimes \ldots \delta^{(k)}\left(t_{q}-\tau_{q}\right), \phi\left(w_{1} \ldots w_{q}\right)\right\rangle \\
& \triangleq\left\langle\delta^{(k)}\left(t_{1}-\tau_{1}\right) \ldots \delta^{(k)}\left(t_{q}-\tau_{q}\right), \mathcal{F} \phi\left(w_{1} \ldots w_{q}\right)\right\rangle \\
& =\left\langle\delta^{(k)}\left(t_{1}-\tau_{1}\right) \ldots \delta^{(k)}\left(t_{q}-\tau_{q}\right), \int_{R} \exp \left[-2 \pi i\left(w_{1} t_{1}+\ldots+w_{q} t_{q}\right)\right] \phi\left(w_{1} \ldots w_{q}\right)\right. \\
& d w_{1} \ldots d w_{q}> \\
& =(-1)^{q k}<\delta\left(t_{1}-\tau_{1}\right) \ldots \delta\left(t_{q}-\tau_{q}\right), \frac{d^{k+k+\ldots k}}{d t_{1}^{k} \ldots d t_{q}^{k}} \int_{R^{q}} \exp \left[-2 \pi\left(w_{1} t_{1}+\ldots+w_{q} t_{q}\right)\right] \phi\left(w_{1} \ldots w_{q}\right) \\
& d w_{1} \ldots d w_{q}> \\
& \begin{aligned}
=\left\langle\delta\left(t_{1}-\tau_{1}\right) \otimes \delta \delta\left(t_{q}-\tau_{q}\right), \int_{R^{q}}\left(2 \pi i w_{1}\right)^{k} \ldots\left(2 \pi i w_{q}\right)^{k} \exp \left[-2 \pi\left(w_{1} t_{1}+\ldots w_{q} t_{q}\right)\right]\right. \\
\left.\phi\left(w_{1} \ldots w_{q}\right) d w_{1} \ldots d w_{q}\right\rangle
\end{aligned} \\
& =\int_{R}\left(2 \pi i w_{1}\right)^{k} \ldots\left(2 \pi i w_{q}\right)^{k} \exp \left[-2 \pi i\left(w_{1} \tau_{1}+\ldots+w_{q} \tau_{q}\right)\right] \phi\left(w_{1} \ldots w_{q}\right) d w_{1} \ldots d w_{q} \\
& =\left\langle\left(2 \pi i w_{1}\right)^{k} e^{-2 \pi i w_{1} \tau_{1}} \cdot\left(2 \pi i w_{2}\right)^{k} e^{-2 \pi i w_{2} \tau_{2}} \ldots\left(2 \pi i w_{q}\right)^{k} e^{-2 \pi i w_{q} \tau} q, \phi\left(w_{1} \ldots w_{q}\right)\right\rangle
\end{aligned}
$$

where $\left(2 \pi i w_{n}\right)^{k} e^{-2 \pi i w_{n} \tau},(1<n<q)$ is being considered as a regular tempered distribution. In summary we have

$$
\left(\delta^{(k)}(t-\tau)\right) \leftrightarrow\left[\begin{array}{c}
\left(2 \pi w_{1}\right)^{k} e^{-2 \pi 1 w_{1} \tau_{1}} \\
\vdots \\
\left(2 \pi i w_{1}\right)^{k}\left(2 \pi i w_{2}\right)^{k} \ldots\left(2 \pi i w_{q}\right)^{k} e^{-2 \pi 1\left[w_{1} \tau_{1}+\ldots+w_{q} \tau_{q}\right]}
\end{array}\right] .
$$


It is clear that any $q^{\prime}$ th entry in expression $(4.6)$ does not belong to $L^{2}\left(R^{q}\right)$ since clearly

$$
\begin{aligned}
& \left|\left(2 \pi i w_{1}\right)^{k} \ldots\left(2 \pi i w_{q}\right)^{k} e^{-2 \pi i\left[w_{1} \tau_{1}+\ldots+w_{q} \tau_{q}\right]}\right|= \\
& {\left[\left(2 \pi w_{1}\right) \ldots\left(2 \pi w_{q}\right)\right]^{k} \text { is not integrable over } R^{q} .}
\end{aligned}
$$

However, the expression given in line (4.5) does belong to the ( $\left.\Gamma^{\mathrm{pB}}\right)$ ' space since

$$
\begin{aligned}
& \|\|\left(\delta^{(k)}(t-\tau)\right)\|\|_{-s B_{m}} \\
& =\sum_{q=0}^{\infty}\left\|F_{q}\right\|_{-m}\left(s B_{m}\right)^{q} q !^{-1 / p} \\
& =\sum_{q=0}^{\infty}\left\|\left(2 \pi i w_{1}\right)^{k} \ldots\left(2 \pi i w_{q}\right)^{k} e^{-2 \pi i\left[w_{1} \tau_{1} \cdots w_{q} \tau_{q}\right]}\right\|_{-m}\left(s B_{m}\right)^{q} q !^{-1 / p} \\
& <\sum_{q=0}^{\infty} 1\left(s B_{m}\right)^{q} q !^{-1 / p}<\infty_{0}
\end{aligned}
$$

EXAMPLE 4.7. In a similar computation it can be shown that

$$
\text { F }\left[\begin{array}{c}
1 \\
\delta \\
\vdots \\
\delta \delta \\
\vdots
\end{array}\right]=\left[\begin{array}{c}
1 \\
1 \\
\vdots \\
1 \\
\vdots
\end{array}\right]
$$

and again the Fourier transform is a member of every set, $\left(\Gamma_{-m}^{p, s B}\right)$. It should be noted that other spaces such as distributions of exponential growth [3] offer some technical achievements that increase the space of Fourier transformable functions. However, we wanted to relate our results to our specialized scales of Frechet spaces developed in Schmeelk [7-10] and Schwartz [15].

\section{REFERENCES}

1. BOGOLUBOV, N.N., LOGUNOV, T.T. and TODOROV, I.T., Introduction to Axiomatic Quantum Field Theory, W.A. Benjamin, Inc., Massachusetts, 1975.

2. CONSTANTINESCU, F., Distributions and their Applications in Physics, Pergammon Pres8, New York, 1980 .

3. FRIEDMAN, A., Generalized Functions and Partial Differential Equations, PrenticeHal1, Englewood Cliff, N.J., 1963.

4. GELFAND, M., SHILOV, G.E., Generalized Functions Volume 2, Academic Press, New York, 1968.

5. LIGHTHILL, M.J., Fourier Analysis and Generalized Functions, Cambridge University Press, England, 1964.

6. ZEMANIAN, A., Distribution Theory and Transform Analysis, McGraw Hill Book Co., New York, 1965.

7. SCHMEELK, J., An Infinite Dimensional Laplacian Operator, J. Differential Equations 36(1) (1980), 74-88.

8. SCHMEELK, J., App11cations of Test Surfunctions, App1. Ana1. 17(3) (1984), 169185 
9. SCHMEELK, J., Infinite Dimensiona1 Parametric Distributions, App1. Ana1. 24 $(1987), 291-317$.

10. SCHMEELK, J., Infinite Dimensional Fock Spaces and Associated Creation and Annihilation Operators, J. Math. Analysis and Applications 134(2) (1988), 111-141.

11. BRACEWELL, R., The Fourier Transform and its Applications, McGraw Hi11, New York, 1986.

12. GONZALEZ, R., WINTZ, P. Digital Image Processing, Addison-Wesley Pub., Co., Massachusetts, 1987.

13. PAPOULIS, A., Signal Analysis, McGraw-Hill Book Co., New York, 1977.

14. CARMICHAEL, R.D., Distributions of Exponential Growth and their Fourler Transforms, Duke Mathematical Journal 40 (1973), 765-783.

15. SCHWARTZ, L., Theorie des Distributions, Hermann, Paris, 1966.

16. COLOMBEAU, J.F., Some Aspects of Infinite-Dimensional Holomorphy in Mathematical Physics, Aspects of Mathematics and its Applications, J.A. Barroso, editor, Elsevier (1986), 253-263.

17. COLOMBEAU, J.F., Different1al Calculus and Holomorphy, North Holland Mathematical Studies, 64 North Holland Pub., Co., New York 1982.

18. COLOMBEAU, J.F., New Generalized Functions and Multiplication of Distributions, North Holland Mathematical studies, 84, North Holland Pub. Co., New York 1984.

19. MANOVKIAN, E.G., Renormalization, Academic Press, New York, 1983.

20. MARKOV, K., Application of Volterra-Weiner Series for Bounding the Overall Conductivity of Heterogeneous Media I. General Procedure, Siam J. Appl. Math. 47 (1987), 836-870.

21. OBERGUGGENBERGER, M., Generalized Solutions to Semilinear Hyperbolic Systems, Mh. Math., 103, (1987), 133-144.

22. OBERGUGGENBERGER, M., Weak Limits of Solutions to Semilinear Hyperbolic Math. Ann., 274 (1986), 599-607.

23. OBERGUGgenberger, M., Products of Distributions, J. fue die Reine Avg. Math. 265 (1986), $1-11$.

24. OPPENHEIM, A., SCHAFER, R.W., Digital Signa1 Processing, Prentice Ha11, Englewood Cliffs, N.J., 1975.

25. COLOMBEAU, J.F., Elementary Introduction to New Generalized Functions, North Holland Mathematical Studies, 113, North Holland Pub. Co., New York, 1985.

26. PERSSON, J., Invariance of the Cauchy Problem for Distributional Differential Equations, Proceedings of the 1987 Generalized Function Conference at Dubrovnik, Yugoslavia, Dellen Pub., Co., 1988.

27. PILIPOVIC, S., Structural Theorems for Periodic U1tradistributions, Proceedings of the A.M.S., 98(2) (1986), 261-266.

28. PILIPOVIC, S., On the Quasiasymptotic Behavior of the Stieltjes Transformation of Distributions, Publication de L'Inst1tut Mathematique, 40 (1986), 143-152.

29. RAJU, C.K., Products and Composition with the Dirac Deta Function, J. Phys. A: Math. Gen. 15 (1982), 381-396.

30. COLOMBEAU, J.F., A Multiplication of Distributions, J. of Math. Ann. and Applications 94(1) (1983), 96-115.

31. COLUMBEAU, J.F. and ROUX, Le, A.Y., Generalized Functions and Products appearing in Equations of Physics, preprint.

32. COOKE, K., WIENER, J., Distributional and Analytical Solutions of Functional Differential Equations, J. of Math. Analysis and Analysis and Applications 98(1) (1984), 111-129.

33. DESPOTOVIC, N. and TAKACI, A., On the Distributional Stieltjes Transformation, Internat. J. Math. and Math Sci. 9(2) (1986), 313.-317.

34. DIRAC, P.A.M., Principles of Quantum Mechanics, Oxford University PressEngland 1967. 
35. SCHWARTZ, L., Impossibilte de la Multiplication des Distributions, Comptes Rendus de $L^{\prime}$ Academie des Science, 239 (1954), 847-848.

36. TODOROV, T.D., Sequential Approach to Colombeaus Theory of Generalized Functions, International Center for Theoretical Physics IC 126 (1987), Trieste, Italy.

37. VELO, G., WIGHTMAN, A.S., Editors, Renormalization Theory, Proceedings of the NATO Advanced Study Institute, International School of Mathematical Physics in Sicily Italy, August 1975, D. Reidel Pub. Co., Boston, 1976.

38. VLAADIMIROV, U.S., DROZZINOV, Y.N., ZAVIALOW, B.I., Taiberian Theorems for Generalized Functions, Kluwer Academic Pub., Boston, Mass., 1988.

39. LIVERMAN, T.P.G., Generalized Functions and Direct Operational Methods, Prentice Ha11, Englewood Cliff., N.J., 1964.

40. ZEMANIAN, A., Generalized Integral Transformations, John Wiley \& Sons, Inc., New York, 1968. 


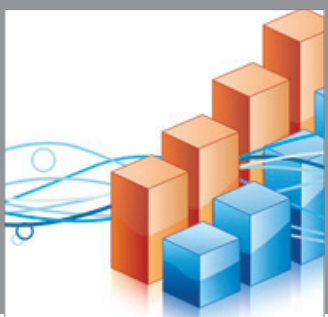

Advances in

Operations Research

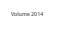

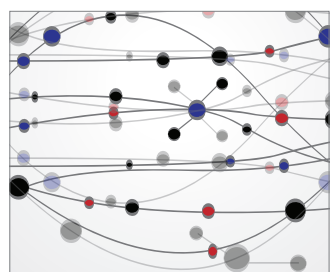

\section{The Scientific} World Journal
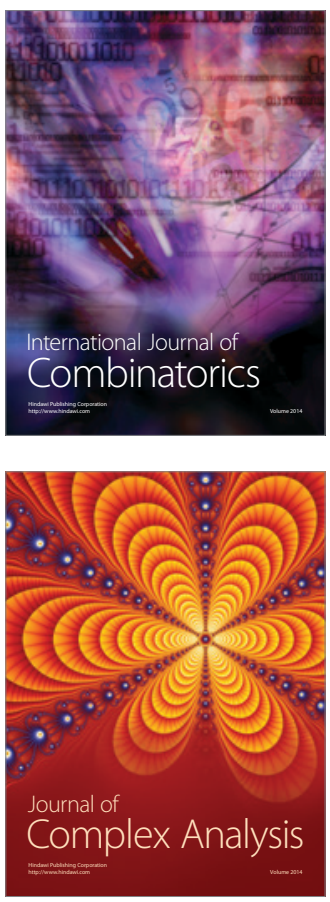

International Journal of

Mathematics and

Mathematical

Sciences
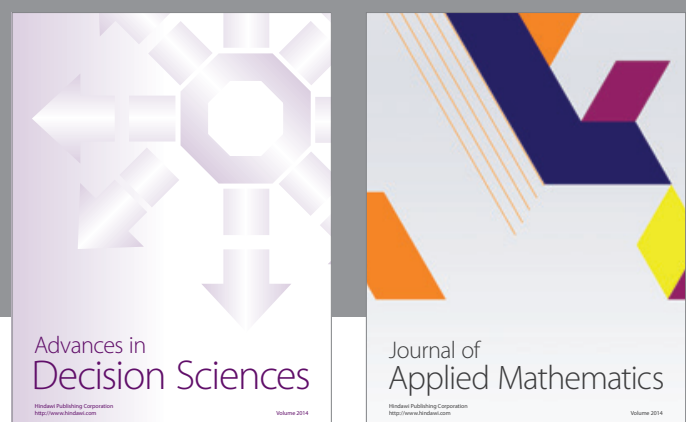

Journal of

Applied Mathematics
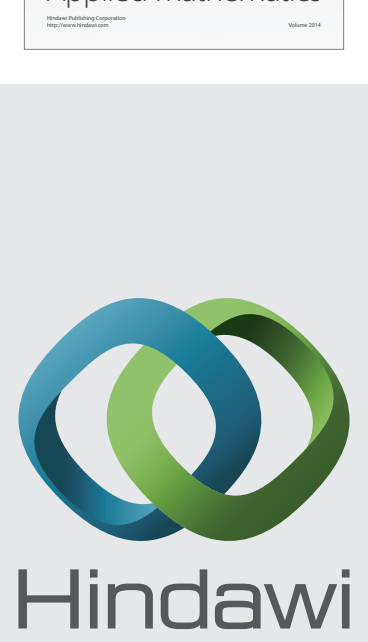

Submit your manuscripts at http://www.hindawi.com
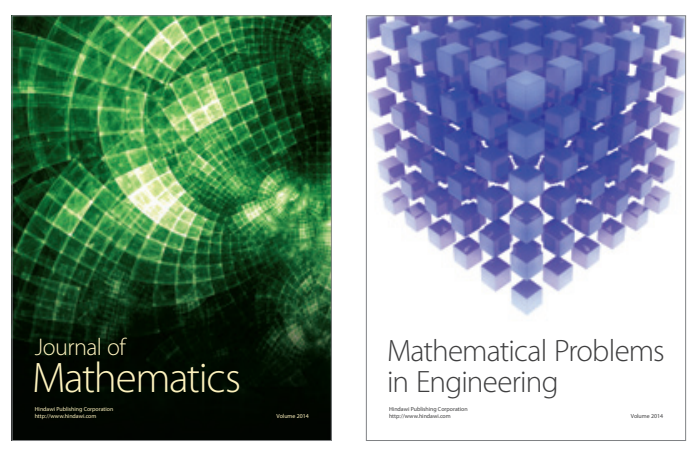

Mathematical Problems in Engineering
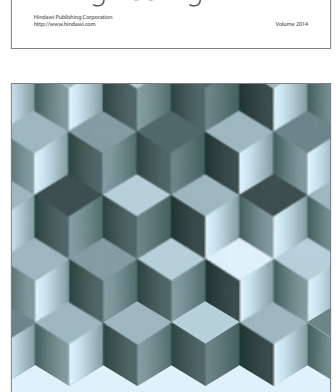

Journal of

Function Spaces
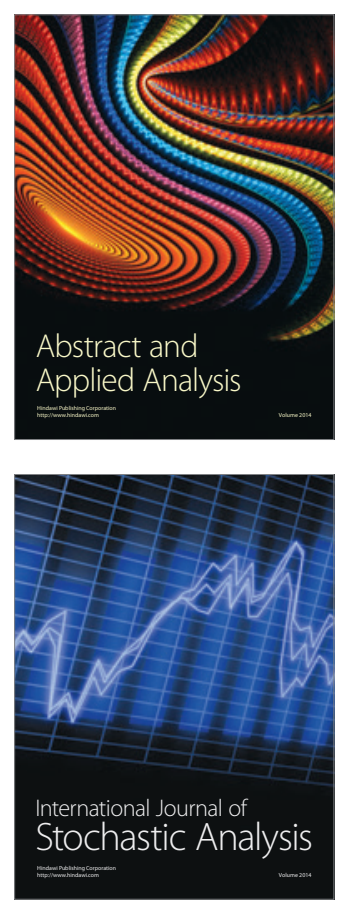

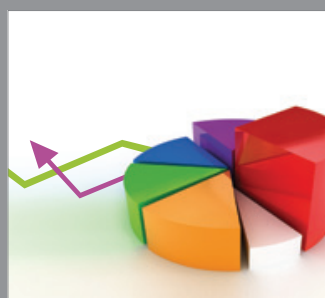

ournal of

Probability and Statistics

Promensencen
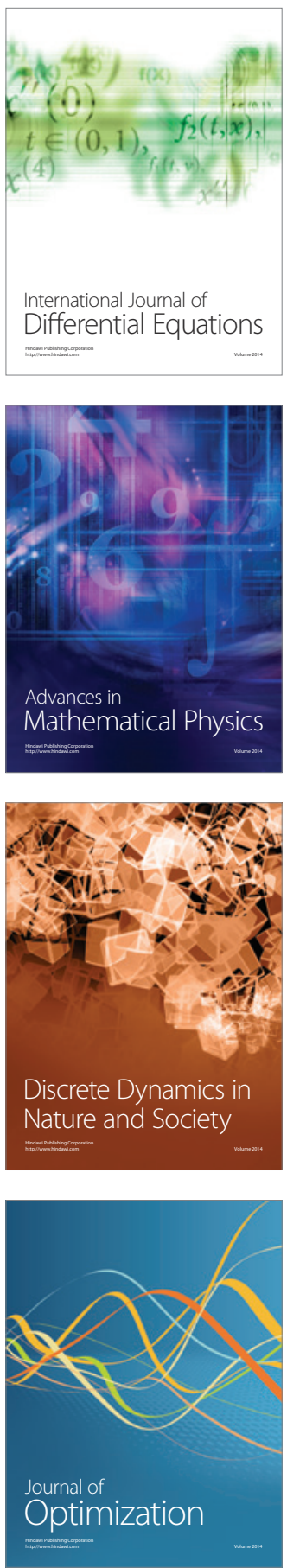